\title{
ON EMBEDDING OF LATTICES BELONGING TO THE SAME GENUS
}

\author{
H. JACOBINSKI
}

ABstract. If $R$ is an order in a semisimple algebra over a Dedekind ring and $M, N$ two $R$-lattices in the same genus, an upper bound for the length of the composition series of $M / N^{\prime}$ for $N^{\prime} \cong N$, is given. This answers a question posed by Rorter.

Let $\mathfrak{o}$ be a Dedekind-ring whose quotient field $k$ is an algebraic number field, $A$ a semisimple algebra over $k$, and $R$ an o-order in $A$. Two $R$-lattices $M, N$ belong to the same genus $\Gamma$ if $M_{p} \cong N_{p}$ for all primes $p$ in $\mathfrak{o}$. In [2] Roitter posed the question whether every $X \in \Gamma$ is isomorphic to a maximal sublattice of $M$. The theorem below answers this question to the affirmative if $A$ is simple, to the negative otherwise.

We will use notations and results from Jacobinski [1], which will be quoted as GD. Let $M$ and $N$ be in the same genus and $N \subset M$. We denote by $l_{R}(M / N)$ the length of a composition series of $M / N$ as $R$-module. Clearly $N$ is a maximal sublattice if and only if $l(M / N)$ $=1$. (For the definition of $\mathscr{L}_{R}^{\prime}$ see GD, Definition 1.3, p. 5.)

TheOREM. Let $\mathfrak{D}$ be a Dedekind ring whose quotient field $k$ is an algebraic number field and $R$ an $\mathrm{o}$-order in the semisimple k-algebra $A=$ $\oplus A_{i}$, with $A_{i}$ simple. Let $M$ be an R-lattice in $\mathfrak{L}_{R}{ }^{\prime}$ and let $t_{M}$ be the number of the algebras $A_{i}$ for which $A_{i} \otimes_{0} M \neq 0$. Then every lattice in the genus $\Gamma(M)$ is isomorphic to a lattice $N \subset M$ such that

$$
l_{R}(M / N) \leqq t_{M} .
$$

Moreover $N$ can be chosen such that the annihilator of $M / N$ is prime to an ideal $d$ in $\mathbf{b}$, given in advance.

Proof. Let $U \neq \varnothing$ be a finite set of primes containing all $p$ such that $R_{p}$ is not a maximal order and also all primes dividing the given ideal $d$ (see GD, p. 11). We embed $R$ in a maximal order $\mathfrak{D}$ and choose a two-sided $\mathfrak{D}$-ideal $\mathfrak{F}$, contained in $R$. For convenience we suppose that $\mathfrak{F}_{p} \neq \mathfrak{D}_{p}$ if and only if $p \in U$. As in GD, let $E(M), E(\mathfrak{D M})$ denote the endomorphism-rings of $M$ and $\mathfrak{D} M$ respectively.

Received by the editors July 31, 1969 .

AMS Subject Classifications. Primary 1640, 1075; Secondary 2080.

Key Phrases. Representation of orders over a Dedekind ring, genus of representation modules, isomorphism classes in a genus, Dirichlet's theorem on arithmetic progressions. 
We replace $\Gamma$ by the subset $S$ of all $N \subset M$, such that the annihilator of $M / N$ is not divisible by any prime of $U$. Every element of $\Gamma$ is isomorphic to some $N \in S$, (GD, Proposition 2.1) and we have to find an $N \in S$ such that $l_{R}(M / N) \leqq t_{M}$. Let $a$ be an integral left $E(\supseteq M)$-ideal such that $\mathfrak{a}_{p}=(1)$ for all $p \in U$. Then $M_{\mathfrak{a}}=M \cap \mathfrak{S}_{\mathfrak{a}}$ is in $S$, and conversely, every element $N$ of $S$ determines a unique ideal a such that $N=M_{\mathfrak{a}}$ (GD, Proposition 21). This means that

$$
\phi: \mathfrak{a} \rightarrow M \cap \mathfrak{D} M_{\mathfrak{a}}
$$

is a 1-1 correspondence between integral $E(\supseteqq M)$-ideals with $\mathfrak{a}_{p}=(1)$, $p \in U$ and the elements of $S$. Since $\phi$ also preserves inclusions we have

$$
l_{R}(M / N)=l_{E(\mathrm{OM})}(E(\preceq M) / \mathfrak{a}) .
$$

The reduced norm $n(\mathfrak{a})$ is an integral ideal in $e_{M} C$, the center of

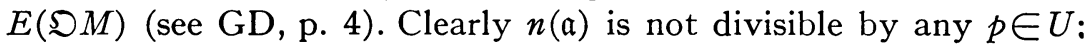
moreover every such ideal in $e_{M} C$ is obtained as $n(\mathfrak{a})$, with $\mathfrak{a}_{p}=(1)$ for all $p \in U$. Now the multiplicativity of the reduced norm implies that

$$
l_{E\left(\bigcirc_{M}\right)}(E(囚 M) / \mathfrak{a})=l_{e_{M} C}\left(e_{M} C / n(\mathfrak{a})\right) .
$$

If we replace $\mathfrak{a}$ by an ideal $\mathfrak{b}$, such that $n(\mathfrak{b}) \in n(\mathfrak{a}) S_{\mathfrak{F}}\left(e_{M}\right)$, then the corresponding lattices $N$ and $V$ are isomorphic (GD, Lemma 2.6 and Theorem 2.2).

Let $e_{i}$ denote the primitive central idempotents in $A$. Then we have

$$
n(\mathfrak{a}) S \mathfrak{F}\left(e_{M}\right)=\underset{e_{\mathfrak{i}} M \neq 0}{\bigoplus} n\left(e_{i} \mathfrak{a}\right) \cdot S_{\mathfrak{F}}\left(e_{i}\right) .
$$

According to the generalized version of Dirichlet's theorem on arithmetic progressions, we can find a prime ideal $p_{i}$ in each $n\left(e_{i} \mathfrak{a}\right) S_{\mathfrak{F}}\left(e_{i}\right)$. If then we choose $\mathfrak{b}$ such that

$$
n(\mathfrak{b})=\underset{e_{i} M \neq 0}{\bigoplus} p_{i}
$$

the corresponding lattice $V$ is isomorphic to $N$ and

$$
l_{R}(M / V)=l_{e_{M} C}\left(e_{M} C / \mathfrak{b}\right)=t_{M},
$$

which completes the proof.

We now turn to the question whether the inequality in the theorem can be improved. For a particular genus $\Gamma$ with $S_{\mathfrak{F}}\left(e_{\Gamma}\right) \neq H_{\Gamma}$, one sees from the proof that this may easily be the case. Moreover we have taken into account only lattices $N \subset M$ such that the annihilator of $M / N$ is prime to $\mathfrak{F}$. Nevertheless the bound given is best possible, if 
no special assumptions are made about the order $R$ or the genus $\Gamma$. To see this choose $A$ such that every maximal order $e_{i} \mathfrak{D}$ has class number $>1$; for this it is sufficient that all $e_{i} C$ have class number $>1$.

Let $e$ be a central idempotent in $A$ and put $M=\mathfrak{D} e$. Then the genus $\Gamma(M)$ consists of all full fractionary ideals $\mathfrak{A}$ in $\mathfrak{D}$. Now choose an integral ideal $\mathfrak{A} \subset \mathfrak{D} e$, such that no $e_{i} \mathfrak{A}$ is principal for $e_{i} \mathfrak{A} \neq 0$. If $\mathfrak{B} \cong \mathfrak{A}$, then each $e_{i} \mathfrak{B} \neq e_{i} \mathfrak{D}$ since the $e_{i} \mathfrak{B}$ are not even principal. This implies that $l_{\mathcal{O}}(M / \mathfrak{B}) \geqq t_{M}$ for every $\mathfrak{B} \cong \mathfrak{A}$. Thus the constant $t_{M}$ cannot in general be improved.

\section{REFERENCES}

1. H. Jacobinski, Genera and decompositions of lattices over orders, Acta. Math. 121 (1968), 1-29.

2. A. V. RoIter, Integer-valued representations belonging to one genus, Izv. Akad. Nauk SSSR Ser. Mat. 30 (1966), 1315-1324; English transl., Amer. Math. Soc. Transl. (2) 71 (1968), 49-59. MR 35 \#4255.

Chalmers University of Technology, Göteborg and UNIVERSITY OF ILLINOIS 